Modern Physics Letters A, Vol. 18, No. 24 (2003) 1661-1671

(C) World Scientific Publishing Company

\title{
DIRAC EQUATION IN THE CONFINING SU(3)-YANG-MILLS FIELD AND THE RELATIVISTIC EFFECTS IN QUARKONIA SPECTRA
}

\author{
YU. P. GONCHAROV \\ Theoretical Group, Experimental Physics Department, State Polytechnical University \\ Sankt-Petersburg 195251, Russia \\ E. A. CHOBAN* \\ Theoretical Physics Department, State Polytechnical University \\ Sankt-Petersburg 195251, Russia
}

Received 5 January 2003

\begin{abstract}
The recently obtained solutions of Dirac equation in the confining SU(3)-Yang-Mills field in Minkowski spacetime are applied to describe the energy spectra of quarkonia (charmonium and bottomonium). The nonrelativistic limit is considered for the relativistic effects to be estimated in a self-consistent way and it is shown that the given effects are extremely important for both the energy spectra and the confinement mechanism.
\end{abstract}

\section{Introductory remarks}

Theory of quarkonium ranks high within the hadron physics as the one of central sources of information about the quark interaction. Referring for more details to the recent up-to-date review, ${ }^{1}$ it should be noted here that at present some generally accepted relativistic model of quarkonium is absent. The description of quarkonium is actually implemented by nonrelativistic manner (on the basis of the Schrödinger equation) and then one tries to include relativistic corrections in one or another way. Such an inclusion is not single-valued and varies in dependence of the point of view for different authors (see, e. g. Ref. ${ }^{2}$ and references therein). It would be more consistent, to our mind, building a primordially relativistic model so that one can then pass on to the nonrelativistic one by the standard limiting transition and, thus, to estimate the relativistic effects in a self-consistent way.

As follows from the main principles of quantum chromodynamics (QCD), the suitable relativistic models for description of relativistic bound states of quarkonium should consist in considering the solutions of Dirac equation in a SU(3)-Yang-Mills field representing gluonic field. The latter should be the so-called confining solution of the corresponding Yang-Mills equations and should model the quark confinement. Such solutions are usually supposed to contain at least one component of the mentioned SU(3)-field linear in $r$, the distance between quarks. Recently in

\footnotetext{
*Deceased.
} 
Ref. $^{3}$ a number of such solutions has been obtained and the corresponding spectrum of the Dirac equation describing the relativistic bound states in this confining SU(3)-Yang-Mills field has been analysed. In this note we should like to apply the results of Ref. ${ }^{3}$ to description of the charmonium and bottomonium spectra. We here solve the inverse problem, i. e. we define the confining gluonic field components in the covariant description (SU(3)-connection) for charmonium and bottomonium employing the experimental data on the mentioned spectra. ${ }^{4}$ As a result, we shall not use any nonrelativistic potentials modelling confinement, for example, of the harmonic oscillator or funnel type, in particular, because the latter ones do not satisfy the Yang-Mills equation while the SU(3)-gluonic field used by us does. In our case the approach is relativistic from the very outset and our considerations are essentially nonperturbative since we shall not use any expansions in the coupling constant $g$ or in any other parameters.

Further we shall deal with the metric of the flat Minkowski spacetime $M$ that we write down (using the ordinary set of local spherical coordinates $r, \vartheta, \varphi$ for spatial part) in the form

$$
d s^{2}=g_{\mu \nu} d x^{\mu} \otimes d x^{\nu} \equiv d t^{2}-d r^{2}-r^{2}\left(d \vartheta^{2}+\sin ^{2} \vartheta d \varphi^{2}\right) .
$$

Besides we have $|\delta|=\left|\operatorname{det}\left(g_{\mu \nu}\right)\right|=\left(r^{2} \sin \vartheta\right)^{2}$ and $0 \leq r<\infty, 0 \leq \vartheta<\pi$, $0 \leq \varphi<2 \pi$.

Throughout the paper we employ the system of units with $\hbar=c=1$, unless explicitly stated otherwise. Finally, we shall denote $L_{2}(F)$ the set of the modulo square integrable complex functions on any manifold $F$ furnished with an integration measure while $L_{2}^{n}(F)$ will be the $n$-fold direct product of $L_{2}(F)$ endowed with the obvious scalar product.

\section{Preliminary considerations}

\subsection{Dirac equation}

To formulate the results of Ref. ${ }^{3}$ needed to us here, let us notice that the relativistic wave function of quarkonium can be chosen in the form

$$
\psi=\left(\begin{array}{c}
\psi_{1} \\
\psi_{2} \\
\psi_{3}
\end{array}\right)
$$

with the four-dimensional spinors $\psi_{j}$ representing $j$-th colour component of quarkonium. The corresponding Dirac equation for $\psi$ may look as follows

$$
\mathcal{D} \psi=\mu_{0} \psi,
$$

where $\mu_{0}$ is a mass parameter while the coordinate $r$ makes sense of the distance between quarks. 
From general considerations the explicit form of the operator $\mathcal{D}$ in local coordinates $x^{\mu}$ on Minkowski manifold can be written as follows

$$
\mathcal{D}=i\left(\gamma^{e} \otimes I_{3}\right) E_{e}^{\mu}\left(\partial_{\mu} \otimes I_{3}-\frac{1}{2} \omega_{\mu a b} \gamma^{a} \gamma^{b} \otimes I_{3}-i g A_{\mu}\right), a<b,
$$

where $A=A_{\mu} d x^{\mu}, A_{\mu}=A_{\mu}^{c} T_{c}$ is a $\mathrm{SU}(3)$-connection in the (trivial) bundle $\xi$ over Minkowski spacetime, $I_{3}$ is the unit matrix $3 \times 3$, the matrices $T_{c}$ form a basis of the Lie algebra of $\mathrm{SU}(3)$ in 3-dimensional space (we consider $T_{a}$ hermitean which is acceptable in physics), $c=1, \ldots, 8, \otimes$ here means tensorial product of matrices, $g$ is a gauge coupling constant. Further, the forms $\omega_{a b}=\omega_{\mu a b} d x^{\mu}$ obey the Cartan structure equations $d e^{a}=\omega_{b}^{a} \wedge e^{b}$ with exterior derivative $d$, while the orthonormal basis $e^{a}=e_{\mu}^{a} d x^{\mu}$ in cotangent bundle and dual basis $E_{a}=E_{a}^{\mu} \partial_{\mu}$ in tangent bundle are connected by the relations $e^{a}\left(E_{b}\right)=\delta_{b}^{a}$. At last, matrices $\gamma^{a}$ represent the Clifford algebra of the corresponding quadratic form $Q_{1,3}=x_{0}^{2}-x_{1}^{2}-x_{2}^{2}-x_{3}^{2}$ in $\mathbb{C}^{2}$. For this we take the following choice for $\gamma^{a}$

$$
\gamma^{0}=\left(\begin{array}{cc}
1 & 0 \\
0 & -1
\end{array}\right), \gamma^{b}=\left(\begin{array}{cc}
0 & \sigma_{b} \\
-\sigma_{b} & 0
\end{array}\right), b=1,2,3,
$$

where $\sigma_{b}$ denote the ordinary Pauli matrices. It should be noted that, in lorentzian case, Greek indices $\mu, \nu, \ldots$ are raised and lowered with $g_{\mu \nu}$ of (1) or its inverse $g^{\mu \nu}$ and Latin indices $a, b, \ldots$ are raised and lowered by $\eta_{a b}=\eta^{a b}=\operatorname{diag}(1,-1,-1,-1)$, so that $e_{\mu}^{a} e_{\nu}^{b} g^{\mu \nu}=\eta^{a b}, E_{a}^{\mu} E_{b}^{\nu} g_{\mu \nu}=\eta_{a b}$ and so on.

We can concretize the Dirac equation (2) for $\psi$ in the case of metric (1). Namely, we can put $e^{0}=d t, e^{1}=d r, e^{2}=r d \vartheta, e^{3}=r \sin \vartheta d \varphi$ and, accordingly, $E_{0}=\partial_{t}$, $E_{1}=\partial_{r}, E_{2}=\partial_{\vartheta} / r, E_{3}=\partial_{\varphi} /(r \sin \vartheta)$. This entails

$$
\omega_{12}=-d \vartheta, \omega_{13}=-\sin \vartheta d \varphi, \omega_{23}=-\cos \vartheta d \varphi .
$$

As for the connection $A_{\mu}$ in bundle $\xi$ then the suitable one should be the confining solution of the Yang-Mills equations

$$
\begin{gathered}
d F=F \wedge A-A \wedge F, \\
d * F=* F \wedge A-A \wedge * F
\end{gathered}
$$

with the exterior differential $d=\partial_{t} d t+\partial_{r} d r+\partial_{\vartheta} d \vartheta+\partial_{\varphi} d \varphi$ in coordinates $t, r, \vartheta, \varphi$ while the curvature matrix (field strentgh) for $\xi$-bundle is $F=d A+A \wedge A$ and * means the Hodge star operator conforming to metric (1). It is clear that (6) is identically satisfied - this is just the Bianchi identity holding true for any connection so that it is necessary to solve only the equations (7).

\subsection{SU(3)-confining connection}

In Ref. ${ }^{3}$ the black hole physics techniques from Refs. ${ }^{5}$ was used to find a set of the confining solutions of Eq. (7). For the aims of the given paper we need one such a solution of Ref. ${ }^{3}$ Let us adduce it here putting $T_{c}=\lambda_{c}$, where $\lambda_{c}$ are the 
Gell-Mann matrices (whose explicit form can be found in Refs. ${ }^{5}$ ) Then the solution in question is the following one

$$
\begin{aligned}
& A_{t}^{3}+\frac{1}{\sqrt{3}} A_{t}^{8}=-\frac{a_{1}}{r}+A_{1},-A_{t}^{3}+\frac{1}{\sqrt{3}} A_{t}^{8}=\frac{a_{1}+a_{2}}{r}-\left(A_{1}+A_{2}\right),-\frac{2}{\sqrt{3}} A_{t}^{8}=-\frac{a_{2}}{r}+A_{2}, \\
& A_{\varphi}^{3}+\frac{1}{\sqrt{3}} A_{\varphi}^{8}=b_{1} r+B_{1},-A_{\varphi}^{3}+\frac{1}{\sqrt{3}} A_{\varphi}^{8}=-\left(b_{1}+b_{2}\right) r-\left(B_{1}+B_{2}\right),-\frac{2}{\sqrt{3}} A_{\varphi}^{8}=b_{2} r+B_{2}
\end{aligned}
$$

with all other $A_{\mu}^{c}=0$, where real constants $a_{j}, A_{j}, b_{j}, B_{j}$ parametrize the solution, and we wrote down the solution in the combinations that are just needed to insert into (2). As is not complicated to see, the solution is a configuration describing the electric Coulomb-like colour field (components $A_{t}$ ) and the magnetic colour field linear in $r$ (components $A_{\varphi}$ ). Also it is easy to check that the given solution satisfy the Lorentz gauge condition that can be written in the form $\operatorname{div}(A)=0$, where the divergence of the Lie algebra valued 1-form $A=A_{\mu}^{c} T_{c} d x^{\mu}$ is defined by the relation

$$
\operatorname{div}(A)=\frac{1}{\sqrt{|\delta|}} \partial_{\mu}\left(\sqrt{|\delta|} g^{\mu \nu} A_{\nu}\right)
$$

\subsection{Dirac equation spectrum and wave functions}

As was shown in Ref. ${ }^{3}$, after inserting the above confining solution into Eq. (2), it admits the solutions of the form

$$
\psi_{j}=e^{i \omega_{j} t} r^{-1}\left(\begin{array}{c}
F_{j 1}(r) \Phi_{j}(\vartheta, \varphi) \\
F_{j 2}(r) \sigma_{1} \Phi_{j}(\vartheta, \varphi)
\end{array}\right), j=1,2,3
$$

with the 2 D eigenspinor $\Phi_{j}=\left(\begin{array}{c}\Phi_{j 1} \\ \Phi_{j 2}\end{array}\right)$ of the euclidean Dirac operator on the unit sphere $\mathbb{S}^{2}$. The explicit form of $\Phi_{j}$ is not needed here and can be found in Refs. ${ }^{6}$ For the purpose of the present paper it is sufficient to know that spinors $\Phi_{j}$ can be subject to the normalization condition

$$
\int_{0}^{\pi} \int_{0}^{2 \pi}\left(\left|\Phi_{j 1}\right|^{2}+\left|\Phi_{j 2}\right|^{2}\right) \sin \vartheta d \vartheta d \varphi=1
$$

i. e., they form an orthonormal basis in $L_{2}^{2}\left(\mathbb{S}^{2}\right)$.

The energy spectrum $\varepsilon$ of quarkonium is given by the relation $\varepsilon=\omega_{1}+\omega_{2}+\omega_{3}$ with

$$
\begin{gathered}
\omega_{1}=\omega_{1}\left(n_{1}, l_{1}, \lambda_{1}\right)=\frac{-\Lambda_{1} g^{2} a_{1} b_{1}+\left(n_{1}+\alpha_{1}\right) \sqrt{\left(n_{1}^{2}+2 n_{1} \alpha_{1}+\Lambda_{1}^{2}\right) \mu_{0}^{2}+g^{2} b_{1}^{2}\left(n_{1}^{2}+2 n_{1} \alpha_{1}\right)}}{n_{1}^{2}+2 n_{1} \alpha_{1}+\Lambda_{1}^{2}} \\
\omega_{2}=\omega_{2}\left(n_{2}, l_{2}, \lambda_{2}\right)=
\end{gathered}
$$


$\frac{-\Lambda_{2} g^{2}\left(a_{1}+a_{2}\right)\left(b_{1}+b_{2}\right)-\left(n_{2}+\alpha_{2}\right) \sqrt{\left(n_{2}^{2}+2 n_{2} \alpha_{2}+\Lambda_{2}^{2}\right) \mu_{0}^{2}+g^{2}\left(b_{1}+b_{2}\right)^{2}\left(n_{2}^{2}+2 n_{2} \alpha_{2}\right)}}{n_{2}^{2}+2 n_{2} \alpha_{2}+\Lambda_{2}^{2}}$
$\omega_{3}=\omega_{3}\left(n_{3}, l_{3}, \lambda_{3}\right)=\frac{-\Lambda_{3} g^{2} a_{2} b_{2}+\left(n_{3}+\alpha_{3}\right) \sqrt{\left(n_{3}^{2}+2 n_{3} \alpha_{3}+\Lambda_{3}^{2}\right) \mu_{0}^{2}+g^{2} b_{2}^{2}\left(n_{3}^{2}+2 n_{3} \alpha_{3}\right)}}{n_{3}^{2}+2 n_{3} \alpha_{3}+\Lambda_{3}^{2}}$

where $\Lambda_{1}=\lambda_{1}-g B_{1}, \Lambda_{2}=\lambda_{2}+g\left(B_{1}+B_{2}\right), \Lambda_{3}=\lambda_{3}-g B_{2}, n_{j}=0,1,2, \ldots$, while $\lambda_{j}= \pm\left(l_{j}+1\right)$ are the eigenvalues of euclidean Dirac operator on unit sphere with $l_{j}=0,1,2, \ldots$ Besides

$$
\alpha_{1}=\sqrt{\Lambda_{1}^{2}-g^{2} a_{1}^{2}}, \alpha_{2}=\sqrt{\Lambda_{2}^{2}-g^{2}\left(a_{1}+a_{2}\right)^{2}}, \alpha_{3}=\sqrt{\Lambda_{3}^{2}-g^{2} a_{2}^{2}} .
$$

Further, the radial part of $(10)$, for instance, for $\psi_{1}$-component, is given at $n_{1}=0$ by

$$
F_{11}=C_{1} A r^{\alpha_{1}} e^{-\beta_{1} r}\left(1-\frac{Y_{1}}{Z_{1}}\right), F_{12}=i C_{1} B r^{\alpha_{1}} e^{-\beta_{1} r}\left(1+\frac{Y_{1}}{Z_{1}}\right),
$$

while at $n_{1}>0$ by

$$
\begin{aligned}
& F_{11}=C_{1} A r^{\alpha_{1}} e^{-\beta_{1} r}\left[\left(1-\frac{Y_{1}}{Z_{1}}\right) L_{n_{1}}^{2 \alpha_{1}}\left(r_{1}\right)+\frac{A B}{Z_{1}} r_{1} L_{n_{1}-1}^{2 \alpha_{1}+1}\left(r_{1}\right)\right], \\
& F_{12}=i C_{1} B r^{\alpha_{1}} e^{-\beta_{1} r}\left[\left(1+\frac{Y_{1}}{Z_{1}}\right) L_{n_{1}}^{2 \alpha_{1}}\left(r_{1}\right)-\frac{A B}{Z_{1}} r_{1} L_{n_{1}-1}^{2 \alpha_{1}+1}\left(r_{1}\right)\right],
\end{aligned}
$$

with the Laguerre polynomials $L_{n_{1}}^{\rho}\left(r_{1}\right), r_{1}=2 \beta_{1} r, \beta_{1}=\sqrt{\mu_{0}^{2}-\left(\omega_{1}-g A_{1}\right)^{2}+g^{2} b_{1}^{2}}$, $A=g b_{1}+\beta_{1}, B=\mu_{0}+\omega_{1}-g A_{1}, Y_{1}=\left[\alpha_{1} \beta_{1}-g a_{1}\left(\omega_{1}-g A_{1}\right)+g \alpha_{1} b_{1}\right] B+g^{2} a_{1} b_{1} A$, $\left.Z_{1}=\left[\left(\lambda_{1}-g B_{1}\right) A+g a_{1} \mu_{0}\right)\right] B+g^{2} a_{1} b_{1} A$. Finally, $C_{1}$ is determined from the normalization condition

$$
\int_{0}^{\infty}\left(\left|F_{11}\right|^{2}+\left|F_{12}\right|^{2}\right) d r=\frac{1}{3}
$$

Analogous relations will hold true for $\psi_{2,3}$, respectively, by replacing $a_{1}, A_{1}, b_{1}, B_{1}, \alpha_{1} \rightarrow$ $a_{2}, A_{2}, b_{2}, B_{2}, \alpha_{3}$ for $\psi_{3}$ and $a_{1}, A_{1}, b_{1}, B_{1}, \alpha_{1} \rightarrow-\left(a_{1}+a_{2}\right),-\left(A_{1}+A_{2}\right),-\left(b_{1}+\right.$ $\left.b_{2}\right),-\left(B_{1}+B_{2}\right), \alpha_{2}$ for $\psi_{2}$ so that $\beta_{2}=\sqrt{\mu_{0}^{2}-\left[\omega_{2}+g\left(A_{1}+A_{2}\right)\right]^{2}+g^{2}\left(b_{1}+b_{2}\right)^{2}}$, $\beta_{3}=\sqrt{\mu_{0}^{2}-\left(\omega_{3}-g A_{2}\right)^{2}+g^{2} b_{2}^{2}}$. Consequently, we shall gain that $\psi_{j} \in L_{2}^{4}\left(\mathbb{R}^{3}\right)$ at any $t \in \mathbb{R}$ and, as a result, the solutions of (10) may describe relativistic bound states of quarkonium with the energy spectrum (12)-(14).

\subsection{Nonrelativistic limit}

Before apllying the above relations to a description of charmonium spectrum let us adduce the nonrelativistic limits (i.e, at $c \rightarrow \infty$ ) for the energies of (12)-(14). The common case is not needed to us in present paper so we shall restrict ourselves to the case of $n_{j}=0,1$ and $l_{j}=0$. Expanding $\omega_{j}$ in $x=\frac{g}{\hbar c}$, we get

$$
\omega_{1}\left(0,0, \lambda_{1}\right)=-x \frac{g a_{1} b_{1}}{\lambda_{1}}+\mu_{0} c^{2}\left[1-\frac{1}{2}\left(\frac{a_{1}}{\lambda_{1}}\right)^{2} x^{2}+O\left(x^{3}\right)\right]
$$




$$
\omega_{1}\left(1,0, \lambda_{1}\right)=-x \frac{\lambda_{1} g a_{1} b_{1}}{\left(1+\left|\lambda_{1}\right|\right)^{2}}+\mu_{0} c^{2}\left[1-\frac{1}{8}\left(\frac{a_{1}}{\lambda_{1}}\right)^{2} x^{2}+O\left(x^{3}\right)\right],
$$

which yields at $c \rightarrow \infty$ (putting $\hbar=c=1$ again)

$$
\omega_{1}\left(0,0, \lambda_{1}\right)=\mu_{0}\left[1-\frac{1}{2}\left(\frac{g a_{1}}{\lambda_{1}}\right)^{2}\right], \omega_{1}\left(1,0, \lambda_{1}\right)=\mu_{0}\left[1-\frac{1}{8}\left(\frac{g a_{1}}{\lambda_{1}}\right)^{2}\right] .
$$

Analogously we shall have

$$
\begin{gathered}
\omega_{2}\left(0,0, \lambda_{2}\right)=-\mu_{0}\left[1-\frac{1}{2}\left(\frac{g\left(a_{1}+a_{2}\right)}{\lambda_{2}}\right)^{2}\right], \omega_{2}\left(1,0, \lambda_{2}\right)=-\mu_{0}\left[1-\frac{1}{8}\left(\frac{g\left(a_{1}+a_{2}\right)}{\lambda_{2}}\right)^{2}\right], \\
\omega_{3}\left(0,0, \lambda_{3}\right)=\mu_{0}\left[1-\frac{1}{2}\left(\frac{g a_{2}}{\lambda_{3}}\right)^{2}\right], \omega_{3}\left(1,0, \lambda_{3}\right)=\mu_{0}\left[1-\frac{1}{8}\left(\frac{g a_{2}}{\lambda_{3}}\right)^{2}\right], \quad
\end{gathered}
$$

where, of course, $\lambda_{j}= \pm 1$ and $\lambda_{j}^{2}=1$.

\section{Relativistic spectrum of charmonium}

Now we can adduce numerical results for constants parametrizing the charmonium spectrum which are shown in Table 1.

Table 1: Gauge coupling constant, mass parameter $\mu_{0}$ and parameters of the confining $\mathrm{SU}(3)$-connection for charmonium.

\begin{tabular}{|c|c|c|c|c|c|c|c|}
\hline$g$ & $\mu_{0}, \mathrm{GeV}$ & $a_{1}$ & $a_{2}$ & $b_{1}, \mathrm{GeV}$ & $b_{2}, \mathrm{GeV}$ & $B_{1}$ & $B_{2}$ \\
\hline 0.618631 & 3.11409 & 0.0102202 & -0.126200 & -3.42927 & -3.91720 & 1.99496 & 2.10796 \\
\hline
\end{tabular}

As for parameters $A_{1,2}$ of solution (8), only the wave functions depend on them while the spectrum does not and within the present paper we consider $A_{1}=A_{2}=0$.

With the constants of Table 1 the present-day levels of charmonium spectrum were calculated with the help of (12)-(14) while their nonrelativistic values with the aid of (20)-(22) according to the following combinations (we use the notations of levels from Ref. ${ }^{4}$ )

$$
\begin{gathered}
\eta_{c}(1 S): \varepsilon_{1}=\omega_{1}(0,0,-1)+\omega_{2}(0,0,-1)+\omega_{3}(0,0,-1), \\
J / \psi(1 S): \varepsilon_{2}=\omega_{1}(0,0,-1)+\omega_{2}(0,0,1)+\omega_{3}(0,0,-1), \\
\chi_{c 0}(1 P): \varepsilon_{3}=\omega_{1}(0,0,-1)+\omega_{2}(0,0,-1)+\omega_{3}(0,0,1), \\
\chi_{c 1}(1 P): \varepsilon_{4}=\omega_{1}(1,0,-1)+\omega_{2}(0,0,1)+\omega_{3}(1,0,-1), \\
\eta_{c}(1 P): \varepsilon_{5}=\omega_{1}(0,0,-1)+\omega_{2}(0,0,1)+\omega_{3}(0,0,1), \\
\chi_{c 2}(1 P): \varepsilon_{6}=\omega_{1}(1,0,1)+\omega_{2}(0,0,-1)+\omega_{3}(0,0,-1),
\end{gathered}
$$




$$
\begin{gathered}
\eta_{c}(2 S): \varepsilon_{7}=\omega_{1}(0,0,1)+\omega_{2}(0,0,-1)+\omega_{3}(1,0,1), \\
\psi(2 S): \varepsilon_{8}=\omega_{1}(0,0,-1)+\omega_{2}(0,0,-1)+\omega_{3}(1,0,1), \\
\psi(3770): \varepsilon_{9}=\omega_{1}(1,0,-1)+\omega_{2}(0,0,-1)+\omega_{3}(0,0,1), \\
\psi(4040): \varepsilon_{10}=\omega_{1}(1,0,1)+\omega_{2}(0,0,-1)+\omega_{3}(0,0,1), \\
\psi(4160): \varepsilon_{11}=\omega_{1}(1,0,1)+\omega_{2}(0,0,1)+\omega_{3}(0,0,1), \\
\psi(4415): \varepsilon_{12}=\omega_{1}(1,0,1)+\omega_{2}(0,0,1)+\omega_{3}(1,0,1) .
\end{gathered}
$$

Table 2 contains experimental values of these levels (from Ref. ${ }^{4}$ ) and our theoretical relativistic and nonrelativistic ones, and also the contribution of relativistic effects in $\%$.

Table 2: Experimental and theoretical charmonium levels

\begin{tabular}{|c|c||c|c|c|}
\hline$\varepsilon_{j}$ & $\begin{array}{c}\text { Experimental } \\
\mathrm{Gev}\end{array}$ & $\begin{array}{c}\text { Relativistic } \\
\mathrm{GeV}\end{array}$ & $\begin{array}{c}\text { Nonrelativistic GeV } \\
\mathrm{GeV}\end{array}$ & $\begin{array}{c}\text { Relativistic contribution } \\
\%\end{array}$ \\
\hline$\varepsilon_{1}$ & 2.97980 & 2.97980 & 3.11255 & 4.45516 \\
\hline$\varepsilon_{2}$ & 3.09688 & 3.09688 & 3.11255 & 0.506165 \\
\hline$\varepsilon_{3}$ & 3.41730 & 3.41730 & 3.11255 & 8.91779 \\
\hline$\varepsilon_{4}$ & 3.51053 & 3.50702 & 3.11967 & 11.0450 \\
\hline$\varepsilon_{5}$ & 3.52614 & 3.53438 & 3.11255 & 11.9350 \\
\hline$\varepsilon_{6}$ & 3.55617 & 3.61609 & 3.11260 & 13.9237 \\
\hline$\varepsilon_{7}$ & 3.59400 & 3.66485 & 3.11967 & 14.8758 \\
\hline$\varepsilon_{8}$ & 3.68600 & 3.71725 & 3.11967 & 16.0759 \\
\hline$\varepsilon_{9}$ & 3.76990 & 3.77773 & 3.11260 & 17.6066 \\
\hline$\varepsilon_{10}$ & 4.04000 & 4.05359 & 3.11260 & 23.2138 \\
\hline$\varepsilon_{11}$ & 4.16000 & 4.17067 & 3.11260 & 25.3694 \\
\hline$\varepsilon_{12}$ & 4.41500 & 4.47062 & 3.11972 & 30.2174 \\
\hline
\end{tabular}

\section{Relativistic spectrum of bottomonium}

Numerical values for the corresponding constants parametrizing the bottomonium spectrum which are shown in Table 3. With the constants of Table 3 the

Table 3: Gauge coupling constant, mass parameter $\mu_{0}$ and parameters of the confining $\mathrm{SU}(3)$-connection for bottomonium.

\begin{tabular}{|c|c|c|c|c|c|c|c|}
\hline$g$ & $\mu_{0}, \mathrm{GeV}$ & $a_{1}$ & $a_{2}$ & $b_{1}, \mathrm{GeV}$ & $b_{2}, \mathrm{GeV}$ & $B_{1}$ & $B_{2}$ \\
\hline 0.172961 & 9.71992 & -0.313881 & -0.188848 & -21.1329 & 34.2764 & 1.48168 & 2.01632 \\
\hline
\end{tabular}

present-day levels of bottomonium spectrum were calculated with the help of (12)(14) while their nonrelativistic values with the aid of (20)-(22) according to the 
following combinations (we use the notations of levels from Ref. ${ }^{4}$ )

$$
\begin{gathered}
\Upsilon(1 S): \varepsilon_{1}=\omega_{1}(0,0,-1)+\omega_{2}(0,0,-1)+\omega_{3}(0,0,-1), \\
\chi_{b 0}(1 P): \varepsilon_{2}=\omega_{1}(0,0,-1)+\omega_{2}(0,0,1)+\omega_{3}(0,0,-1), \\
\chi_{b 1}(1 P): \varepsilon_{3}=\omega_{1}(0,0,-1)+\omega_{2}(0,0,-1)+\omega_{3}(0,0,1), \\
\chi_{b 2}(1 P): \varepsilon_{4}=\omega_{1}(1,0,1)+\omega_{2}(1,0,1)+\omega_{3}(0,0,-1), \\
\Upsilon(2 S): \varepsilon_{5}=\omega_{1}(0,0,-1)+\omega_{2}(1,0,1)+\omega_{3}(0,0,1), \\
\chi_{b 0}(2 P): \varepsilon_{6}=\omega_{1}(1,0,1)+\omega_{2}(1,0,-1)+\omega_{3}(0,0,1), \\
\chi_{b 1}(2 P): \varepsilon_{7}=\omega_{1}(1,0,-1)+\omega_{2}(0,0,-1)+\omega_{3}(0,0,1), \\
\chi_{b 2}(2 P): \varepsilon_{8}=\omega_{1}(0,0,1)+\omega_{2}(0,0,-1)+\omega_{3}(1,0,-1), \\
\Upsilon(3 S): \varepsilon_{9}=\omega_{1}(1,0,1)+\omega_{2}(1,0,1)+\omega_{3}(0,0,1), \\
\Upsilon(4 S): \varepsilon_{10}=\omega_{1}(0,0,1)+\omega_{2}(1,0,-1)+\omega_{3}(1,0,1), \\
\Upsilon(10860): \varepsilon_{11}=\omega_{1}(0,0,-1)+\omega_{2}(1,0,1)+\omega_{3}(1,0,-1), \\
\Upsilon(11020): \varepsilon_{12}=\omega_{1}(0,0,1)+\omega_{2}(0,0,1)+\omega_{3}(1,0,1) .
\end{gathered}
$$

Table 4 contains experimental values of these levels (from Ref. ${ }^{4}$ ) and our theoretical relativistic and nonrelativistic ones, and also the contribution of relativistic effects in $\%$.

Table 4: Experimental and theoretical bottomonium levels

\begin{tabular}{|c|c||c|c|c|}
\hline$\varepsilon_{j}$ & $\begin{array}{c}\text { Experimental } \\
\mathrm{Gev}\end{array}$ & $\begin{array}{c}\text { Relativistic } \\
\mathrm{GeV}\end{array}$ & $\begin{array}{c}\text { Nonrelativistic } \\
\mathrm{GeV}\end{array}$ & $\begin{array}{c}\text { Relativistic contribution } \\
\%\end{array}$ \\
\hline$\varepsilon_{1}$ & 9.46037 & 9.46037 & 9.73716 & 2.92574 \\
\hline$\varepsilon_{2}$ & 9.8598 & 9.8598 & 9.73716 & 1.24391 \\
\hline$\varepsilon_{3}$ & 9.8919 & 9.8919 & 9.73716 & 1.56436 \\
\hline$\varepsilon_{4}$ & 9.9132 & 9.95542 & 9.72034 & 2.36137 \\
\hline$\varepsilon_{5}$ & 10.02330 & 10.0427 & 9.70960 & 3.31702 \\
\hline$\varepsilon_{6}$ & 10.2321 & 10.2359 & 9.72034 & 5.03641 \\
\hline$\varepsilon_{7}$ & 10.2552 & 10.2521 & 9.74790 & 4.91837 \\
\hline$\varepsilon_{8}$ & 10.2685 & 10.2611 & 9.74104 & 5.06841 \\
\hline$\varepsilon_{9}$ & 10.3553 & 10.3870 & 9.72034 & 6.41781 \\
\hline$\varepsilon_{10}$ & 10.5800 & 10.6315 & 9.71349 & 8.64370 \\
\hline$\varepsilon_{11}$ & 10.8650 & 10.8536 & 9.71349 & 10.5041 \\
\hline$\varepsilon_{12}$ & 11.019 & 11.0312 & 9.74104 & 11.6955 \\
\hline
\end{tabular}




\section{Physical interpretation}

\subsection{Role of the colour magnetic field}

The results obtained allow us to draw a number of conclusions. As is seen from Tables 2,4, relativistic values are in good agreement with experimental ones while nonrelativistic ones are not. The contribution of relativistic effects can amount to tens per cent and they cannot be considered as small. The physical reason of it is quite clear. Really, we have seen in nonrelativistic limit [see the relations (19)(22)] that parameters $b_{1,2}, B_{1,2}$ [see Eq. (8)] of linear interaction between quarks vanish under this limit and nonrelativistic spectrum is independent of them and is practically getting the pure Coulomb one. As a consequence, the picture of linear confinement for quarks should be considered as essentially relativistic one while the nonrelativistic limit is only a rather crude approximation. In fact, as follows from exact solutions of SU(3)-Yang-Mills equations of (8), the linear interaction between quarks is connected with colour magnetic field that dies out in the nonrelativistic limit, i.e. for static quarks. Only for the moving rapidly enough quarks the above field will appear and generate linear confinement between them. So the spectrum will depend on both the static Coulomb colour electric field and the dynamical colour magnetic field responsible for the linear confinement for quarks which is just confirmed by the relations (12)-(14). For bottomonium the mentioned effects are smaller than for charmonium which well corresponds to physics in question - in the former case quarks are more massive and relativistic effects should be smaller. Also one can say in other words that colour magnetic field splits the primordially nonrelativistic spectrum into some fine structure as, for example, the Seemann effect does in atomic physics. But, unlike the latter case, for quarkonia these corrections are not small.

\subsection{Specification of the wave function form}

One can notice that the form of wave functions (16)-(17) permits to consider, for instance, the quantity $1 / \beta_{1}$ to be a characteristic size of quarkonium. Under the circumstances, if calculating $1 / \beta_{1}$ in both relativistic and nonrelativistic cases then one can obtain, for example, for the charmonium $\eta_{c}(2 S)$-level that relativistic size will be of order $r \sim 1 / \beta_{1} \sim 0.938854 \cdot 10^{-14} \mathrm{~cm}$ while nonrelativistic one (i.e. if calculating $\beta_{1}$ at $b_{1}=0$ ) would be of order $r_{0} \sim 0.101539 \cdot 10^{-11} \mathrm{~cm}$, i. e., $r_{0} / r \sim 108>>1$. Analogously, e. g., for the bottomonium $\Upsilon(1 S)$-level the conforming quantities will be: $r \sim 0.618867 \cdot 10^{-14} \mathrm{~cm}, r_{0} \sim 0.379148 \cdot 10^{-13}$ $\mathrm{cm}, r_{0} / r \sim 6>1$. This additionally points out the importance of relativistic effects for confinement.

\subsection{Comparison with the potential approach}

One should say a few words concerning the nonrelativistic potential models often used in quarkonium theory. ${ }^{7}$ The potentials between quarks here are usually 
modelled by those of harmonic oscillator or of funnel type (i. e. of the form $\alpha / r+\beta r$ with some constants $\alpha$ and $\beta$ ). It is clear, however, that from the QCD point of view the interaction between quarks should be described by the whole $\mathrm{SU}(3)$-connection $A_{\mu}=A_{\mu}^{c} T_{c}$, genuinely relativistic object, the nonrelativistic potential being only some component of $A_{t}^{c}$ surviving in the nonrelativistic limit at $c \rightarrow \infty$. As is easy to show, however, the $\mathrm{SU}(3)$-connection of form $A_{t}^{c}=B r^{\gamma}$, where $B$ is a constant, may be solution of the Yang-Mills equations (7) only at $\gamma=-1$, i. e. in the Coulomblike case. As a result, the potentials employed in nonrelativistic approaches do not obey the Yang-Mills equations. The latter ones are essentially relativistic and, as we have seen, the components linear in $r$ of the whole $A_{\mu}$ are different from $A_{t}$ and related with colour magnetic field vanishing in the nonrelativistic limit. That is why the nonrelativistic potential approach seems to be inconsistent. Our approach uses only the exact solutions of the Yang-Mills equations as well as in atomic physics the interaction among particles (e. g. the electric Coulomb one) is always the exact solution of the Maxwell equations (the particular case of the Yang-Mills equations).

\section{Concluding remarks}

The calculations of the present paper can be extended. Indeed we have the explicit form (10) for the relativistic wave functions of quarkonium that may be applied to analysis of the quarkonium radiative decays and electromagnetic transitions. Unfortunately in connection with death of E. Choban the work in this direction is not yet completed but the first author hopes to discuss the mentioned questions elsewhere.

\section{Acknowlegments}

The work of Goncharov was supported in part by the Russian Foundation for Basic Research (grant no. 01-02-17157).

\section{To the memory of E. A. Choban}

In the process of working at the present paper it happened big misfortune -late in May 2002 E. Choban suddenly died so the paper has been finished without him. I tried to keep everything that we could discuss together. Let the given paper be the last tribute to the remarkable man and enthusiast of hadronic physics who was Enver Choban.

\section{References}

1. B. Grinstein Int. J. Mod. Phys. A15, 461 (2000).

2. S. Louise, J.-J. Dugne and J.-F. Mathiot Phys. Lett. B472, 357 (2000).

3. Yu. P. Goncharov, Mod. Phys. Lett. A16, 557 (2001).

4. Particle Data Group: C. Caso et.al. Euro. Phys. J. C3, 580 (1998). 
5. Yu. P. Goncharov, Nucl. Phys. B460, 167 (1996);

Int. J. Mod. Phys. A12, 3347 (1997);

Pis'ma v ZhETF 67, 1021 (1998);

Mod. Phys. Lett. A13, 1495 (1998).

6. Yu. P. Goncharov, Pis'ma v ZhETF 69, 619 (1999); Phys. Lett. B458, 29 (1999).

7. H. Grosse and A. Martin, Particle Physics and the Schrödinger Equation (Cambridge University Press, Cambridge, 1997). 